\title{
Psychosocial Factors of Diseases: A Reflection from General Medicine
}

\section{Turabian JL*}

Specialist in Family and Community Medicine, Health Center Santa Maria de Benquerencia, Spain

*Corresponding author: Jose Luis Turabian, Specialist in Family and Community Medicine, Health Center Santa Maria de Benquerencia Toledo, Spain, Email: jturabianf@hotmail.com

\section{Research Article \\ Volume 4 Issue 1}

Received Date: February 21, 2020

Published Date: March 28, 2020

DOI: $10.23880 /$ eij-16000139

\section{Abstract}

A psychosocial factor is a measure that relates social phenomena and social contexts with pathophysiological changes. Doctors can recognize the psychosocial influence on patients' health, but we lack the understanding and the tools to incorporate it into a useful way in the diagnostic and treatment. It is a very important issue because all health problems are biopsychosocial. There is an interaction between the different levels: the social determines and modifies the psychological, and the psychological determines and modifies the biological; and vice versa. Every disease takes, in different proportions, the three levels. The psychosocial aspects of diseases are the factors that intervene in the patient's ways of reacting to the disease: their thoughts, emotions, behaviors and habitual bodily sensations when people face a medical diagnosis, and play a role in the expression of the symptoms and diseases. Take into account the "psychosocial" and address the psychosocial in the practice of general medicine, means listening to the patient using a criterion of "simultaneity": listen to the psychological and social information in the patient's complaint (including the symbolic aspects of the story; for example, those that may be related to the metaphoric use of the symptom), while working with the development of organic aspects. Doctor-patientpsychosocial context relationship is the main tool of the general practitioner in the diagnosis and treatment of all health problems in all patients. In addition, it should be remembered that when one knows how to study biological factors and their experiences in a deep and authentic way, one can also enter or at least find the psychosocial spheres of health problems. Thus, Osler's aphorism is confirmed "it is much more important to know what kind of patient you have a disease than what sort of disease a patient has."

Keywords: Biopsychosocial; General Practice; Medical Practice; Clinical Medicine; Social Determinants of Health; Culture; Emotion; Relationships; Society; Diseases

Abbreviations: GP: General Practitioner.

\section{Introduction}

Doctors tend to see the social world as a messy and impenetrable black box: we can recognize its influence on patients' health, but we lack the understanding and the tools to incorporate it in a useful way in the diagnostic and treatment. But the social sciences of health and medicine provide such tools, theories and methods to understand it [1]. The identification and management of the physical signs and symptoms of (chronic) illnesses has improved, but the psychosocial burden is often not recognized and neglected [2].

What is a psychosocial factor? It is a measure that 


\section{Epidemiology International Journal}

potentially relates to psychological phenomena and social contexts with pathophysiological changes. Psychosocial problems constitute between $3-13 \%$ of the main reasons for consultation with general practitioner (GP), and probably in same proportion in other specialists, but they are present in a much larger proportion of patients. Some study has found them between $20-60 \%$ of the patients who consult for an organic reason, depending on the type of problem. Or, perhaps, is not it more accurate (and reasonable) to think that they can be found in $100 \%$ of patients? [3].

Chronic diseases (cancer, cardiovascular diseases, rheumatic diseases, etc.) carry important psychological and social consequences (socioeconomic variables, culture / ethnicity, processes related to gender, interpersonal relationships, personality attributes, cognitive assessments, and coping processes) that require significant psychological adjustment. The experience of chronic disease requires adaptation in multiple areas of life. Heterogeneity in adjustment is apparent between individuals and throughout the course of the disease trajectory $[4,5]$.

In this scenario, this small article aims to contribute to the reflection and knowledge of psychosocial factors from the point of view of general medicine. A personal point of view, or personal opinion, based on own experience, was used; that is, the posture, consideration, attitude, and opinion that the author adopts about psychosocial factors of diseases. Therefore, this personal point of view to obtain the information and to analyze it, is subject to the subjectivity of the author. However, trying to achieve a broad and contextualized perspective, it is intended to minimize this subjectivity of the gaze or focus on the subject.

\section{Discussion}

It can be said that all health problems are biopsychosocial. The symptoms and diagnoses of the disease (cough, dyspnea, hemorrhage, pain, palpitations, epigastric burning, vomiting, diarrhea..., cancer, myocardial infarction, asthma, ulcer ...) symbolize certain psychosocial aspects in people (disability, death, social isolation, anguish, cultural rejection...). In diseases of "biological or organic" origin, psychosocial causes are involved in their etiopathogenesis, evolution and management, and psychosocial symptoms can occur frequently. In "functional or psychosocial" diseases, "somatic" symptoms often accompany psycho-sociopathological manifestations [6]. All health problems are individual, group and community at the same time. In the ethiopathogenesis, evolution and treatment of all health problems are involved biological factors, but also psychosocial (contextual, relational, community) [7]. The disease supposes an alteration or dysfunction of the communication relationships between actors and contexts (human beings, perceptions, environments, etc.) [8].

In the development of living matter, over the course of millions of years, life progressively took more specialized forms ascending the zoological scale: protozoos-mollusksfish-reptiles-birds-mammals-primates. Primates make the leap from the biological to the psychological, but more elaborate functions that capture the environment as a set of objects and phenomena with value and meaning, determined by the relationship of these objects and phenomena with their needs and with previous experiences stored in your memory, have not yet appeared. When the primate becomes a man, by grouping and working for collective purposes, the social level is reached.

There is an interaction between the different levels: the social determines and modifies the psychological, and the psychological determines and modifies the biological; and vice versa. Every disease takes, in different proportions, the three levels (Figure 1).

FIGURE 1. THE INTERACTION BETWEEN THE SOCIAL, THE PSYCHOLOGICAL AND THE BIOLOGICAL

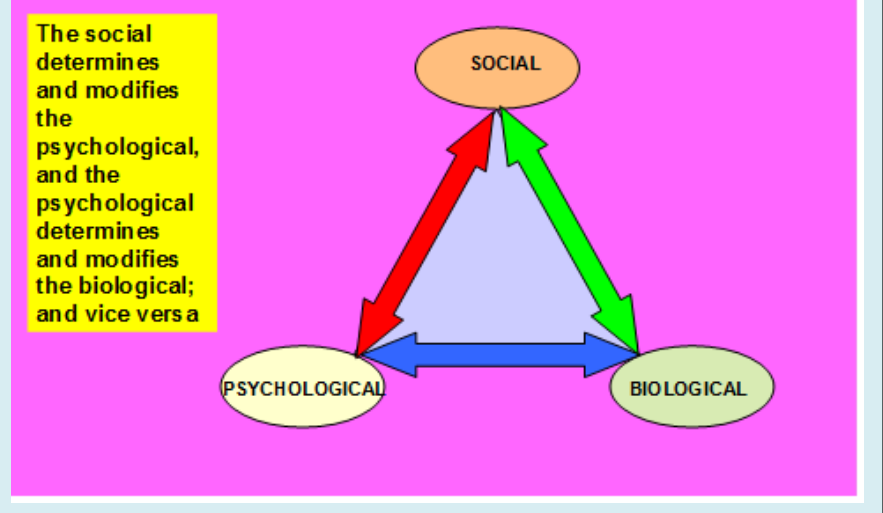

Figure 1: The Interaction between the Social, the Psychological and the Biological.

- A biological (genetic) affection, such as diabetes mellitus, is expressed mainly in the biological level, but the limitations and risks that it implies affect the psychic and social levels of the sufferer.

- A psychic affection is also expressed at the somatic level (constipation, weight loss), and affects the social by reducing the patient's work capacity and their human relationships.

- A social problem (a war ...), affects both the psychological and somatic level of man.

The person is a biopsychosocial unit: Has biological needs ("drink water"); Have psychological needs ("feel 


\section{Epidemiology International Journal}

respected"); He has social needs ("dressing according to fashion"). The impossibility of satisfying these needs can produce imbalances in different degrees.

The psychosocial aspects of diseases are the factors that intervene in the patient's ways of reacting to the disease: their thoughts, emotions, behaviors and habitual bodily sensations when people face a medical diagnosis, and play a role in the expression of the symptoms and diseases. And so, have implications in people's lives.

The personal characteristics in a disease situation create different social and relational contexts: consequences of disability, ethnicity, sex, gender, age, temperament..., behaviour, attitudes. And also, there are consequences of social and relational contexts on individuals: the family, school and community on the individual (on development, welfare, culture, beliefs, values..., isolation in remote rural areas, etc.) $[9,10]$.

That is to say, on the one hand, the patient's condition influences the behaviour of the societies in which they live, but the rejection or acceptance of these also modulates the personal experience of the disease. The perception of the disease has been shown to be useful in predicting many psychosocial and clinical outcomes in patients with different diseases $[11,12]$.

The interpretation and meaning that the individual attributes to the symptoms or discomforts are influenced by different variables such as previous personal experiences with the symptom, family experiences or of other significant actors, models learned, beliefs and social norms. All these factors not only influence the perception of the individual, but at the same time they shape the reaction to the symptoms as a result of their cognitive process. This social label can imply stigma: a social construction that defines people in terms of a distinctive feature or brand, and devalues that person as a consequence. Threats to bodily integrity, or to survival, give rise to fears and anxiety. Patients with high levels of anxiety have difficulty making decisions, and sometimes these are made against their own interests as an attempt to reduce fear. Thus, surgery can be rejected, voluntary discharge from the hospital can be requested, or the doctor can be tricked into important issues (for example, not mentioning a lump in the breast). It can also produce changes in roles in the family, changes in communication, anger, guilt, frustration, disability, changes in habits, including alterations in sexuality $[13,14]$.

Society is made up of human beings and it is clear that the disease, as a personal affection, frequently entails repercussions in both directions. On the one hand, the condition of patients influences the behavior of the societies in which they live, but the rejection or acceptance of these also modulates the personal experience of the disease. In addition, the disease is a system of interpretation. The patient's experience is opened during the clinical consultation. The disease has symbolic and sociocultural meanings for the patient and the doctor. The patient's response to the psychological stress that evokes the medical illness and the extension of the regression that occurs as a consequence of the diseases modifies the quality of the doctor-patient relationship. Therefore, the doctor-patient relationship may be different, for example, in the cardiovascular patient, the hypertensive patient, the asthmatic patient, the digestive patient, the patient in psychiatric diseases, in the digestive system, in the endocrine problems, with an incurable disease, with hematological diseases, with cancer, or with AIDS, among others [14-18]. Table 1 presents a list of some of health and disease determinants [19-22].

\begin{tabular}{|c|l|}
\hline \multicolumn{2}{|c|}{ Some Psychosocial Determinants of Health and Disease } \\
\hline 1 & -Disability \\
\hline 2 & -The patient's previous psychological functioning \\
\hline 3 & -The severity of the deficit of the psychological function associated with the disease \\
\hline 4 & -Residual skills, adaptation and coping with functional deficits \\
\hline 5 & -Ethnicity \\
\hline 6 & -Sex and gender \\
\hline 7 & -Age \\
\hline 8 & -Temperament (for example, type A personality / hostility), behavior, attitudes \\
\hline 9 & -Depression and anxiety \\
\hline 10 & -Professional/job psychosocial characteristics \\
\hline 11 & -Social support/social capital \\
\hline 12 & -Family structure (family stress, roles and relationships, family resources ...) and the family life cycle \\
\hline
\end{tabular}




\section{Epidemiology International Journal}

\begin{tabular}{|l|l|}
\hline 13 & -School, teachers, \\
\hline 14 & -Media (social expectations..., social demands ..., culture ...) \\
\hline 15 & -Culture, beliefs, values \\
\hline 16 & -Isolation in remote rural areas \\
\hline 17 & -Time ("time as a commodity" and "time as a duty"), for example, as a reason for poor dietary habits \\
\hline 18 & -Doctor-patient relationship (trust) \\
\hline 19 & -The partners, colleagues \\
\hline 20 & -Economic level \\
\hline 21 & -Roles and social activities \\
\hline 22 & -Resilience, empowerment \\
\hline
\end{tabular}

Table 1: Psychosocial Determinants Of Health And Disease.

\section{Conclusion}

In short, "the psychosocial" in the practice of general medicine, in GP consultation, means listening to the patient using a criterion of "simultaneity". Listen to the psychological information in the patient's complaint (including the symbolic aspects of the story, for example, those that may be related to the metaphoric use of the symptom), while working with the development of organic aspects. Consequently, doctorpatient-psychosocial context relationship is the main tool of the GP in the diagnosis and treatment of all health problems in all patients. The model of doctor-patient-psychosocial context relationship should be essentially the same in all cases, in both "biological" and "psychosocial" diseases, because all health problems are always bio-psycho-social.

For example, in all cases of hypertension (to mention a very frequent health problem in general medicine and that is accepted as a disease with a "biological" cause), the GP should explore: the patient's attitude towards the disease, the patient's information on the disease, the presence of environmental factors that affect its evolution, etc. Or, to cite another example, in cases of carpal tunnel syndrome, the most common entrapment neuropathy of the upper limb, de GP should explore the association with psychosocial factors [23]. In addition, it should be remembered that when one knows how to study biological factors and their experiences in a deep and authentic way, one can also enter or at least find the psychosocial spheres of health problems.

That is, in all health problems must be exploring the psychosocial factors: contexts, actors, resources. From the concept of health (for example, according to the WHO definition: "Not only the absence of symptoms, but the enjoyment by a person of the physical, mental and social well-being in their environment"), the importance of patientpsychosocial environment relationship is concluded. Thus, Osler's aphorism is confirmed "it is much more important to know what kind of patient you have a disease than what sort of disease a patient has" [24].

\section{References}

1. Stonington SD, Holmes SM, Hansen H, Greene JA, Wailoo KA, et al. (2018) Case Studies in Social Medicine Attending to Structural Forces in Clinical Practice. N Engl J Med 379: 1958-1961.

2. Turner J, Kelly B (2000) Emotional dimensions of chronic disease. West J Med 172(2): 124-128.

3. Turabian JL (2017) Psychosocial Factors and Organic Disease: Two Sides of the Same Coin. Chronicle of Medicine and Surgery 1(1): 44-46.

4. Stanton AL, Revenson TA, Tennen H (2007) Health Psychology: Psychological Adjustment to Chronic Disease. Annu Rev Psychol 58: 565-592.

5. Benedicto AS (2012) Biological and cultural dimension as asymptotes thrill: an actual possibility in the understanding of emotion. Aten Primaria 44(5): 248249.

6. Wileman L, May C, Chew-Graham CA (2002) Medically unexplained symptoms and the problem of power in the primary care consultation: a qualitative study. Fam Pract 19(2): 178-182.

7. Turabian JL, Perez Franco B (2006) The Doctor with Three Heads. Aten Primaria 38(10): 570-573.

8. Turabian JL, Perez Franco B (2007) Big Mysteries. Can You See the Loch Ness Monster? The Biopsychosocial Model and Community Activities. Aten Primaria 39(5): 261-264.

9. Bowes JM, Hayes A (1999) Children, families, and communities. Contexts and consequences. Victoria, Australia: Oxford University Press. 


\section{Epidemiology International Journal}

10. Sewitch MJ, Leffondré K, Dobkin PL (2004) Clustering patients according to health perceptions: relationships to psychosocial characteristics and medication nonadherence. J Psychosom Res 56(3): 323-332.

11. Chilcot J (2012) The importance of illness perception in end-stage renal disease: associations with psychosocial and clinical outcomes. Semin Dial 25(1): 59-64.

12. Woloshynowych M, Valori R, Salmon P (1998) General practice patients' beliefs about their symptoms. Br J Gen Pract 48(426): 885-889.

13. Markus AC, Murray Parkes C, Tomson P, Johnston M (1991) Psychological problems in general practice. Oxford: Oxford University Press.

14. Turabián JL, Pérez-Franco B (2014) Journey to what is essentially invisible: Pysochosocial aspects of disease. Semergen $40(2): 65-72$.

15. Gallon RL (1982) The psichosomatic approach to illness. New York: Elsevier biomedical.

16. Conroy RM, Teehan M, Siriwardena R, Smyth O, McGee HM, et al. (2002) Attitudes to doctors and medicine: The effect of setting and doctor-patient relationship. $\mathrm{Br} \mathrm{J}$ Health Psychol 7(1): 117-125.
17. Turabian JL (2019) Doctor-Patient Relationships: A Puzzle of Fragmented Knowledge. J Family Med Prim Care Open Access 3: 128.

18. Turabian JL (2019) Doctor-patient relationship according the psychosocial aspects of diseases in general medicine. Associative Journal of Health Sciences.

19. Helman CG (1994) Culture, health and illness. Oxford: Butterworth-Heinemann Ltd.

20. Madsen W, Judd J, Williams SL, McKenzie F, Deagon J, et al. (2020) Time as a Social and Environmental Determinant of Health for Rural Women. Health Educ Behav.

21. Turabian JL , Perez Franco B (2001) Community Activities in Family Medicine and Primary Care. Díaz de Santos.

22. Kimbal CP (1981) The biopsychosocial approach to the patient. Baltimore: Williams \& Wilkins.

23. Mansfield M, Thacker M, Sandford F (2018) Psychosocial Risk Factors and the Association With Carpal Tunnel Syndrome: A Systematic Review. Hand 13(5): 501-508.

24. John M (2013) From Osler to the cone technique. HSR Proc Intensive Care Cardiovasc Anesth 5(1): 57-58. 